\title{
Placental Glucose Transporter Gene Expression and Metabolism in the Rat
}

\author{
Jian Zhou and Carolyn A. Bondy \\ Developmental Endocrinology Branch, National Institute of Child Health and Human Development, \\ National Institutes of Health, Bethesda, Maryland 20892
}

\begin{abstract}
In situ hybridization was used to evaluate patterns of gene expression for glucose transporters 1-4 (GT1-4) in the rat uteroplacenta from the time of implantation through term, and in vivo regional placental glucose metabolism was measured by ${ }^{14} \mathrm{C}$-labeled 2-deoxyglucose uptake. GT1 mRNA was highly abundant and GT3 was barely detected in the postimplantation decidual reaction. GT1 and 3 mRNAs were colocalized in the labyrinthine syncitiotrophoblast layer of the chorioallantoic placenta, which forms the membranous barrier between maternal and fetal circulations. The level of labyrinthine GT3 mRNA showed no change from midgestation through term; however, the volume of the labyrinth and hence total GT 3 gene expression increased greatly during this period. Labyrinthine GT1 mRNA levels, in contrast, showed significant diminution near term. GT1 mRNA was also localized in the placental growth plate, or junctional zone, where it was most abundant during the period of rapid placental growth and was decreased at term. Placental glucose metabolism, as reflected by steady-state 2-deoxyglucose uptake, was highest in the junctional zone during the rapid growth phase during midgestation, and decreased significantly at term, in parallel with GT1 gene expression. These findings suggest that GT1 is responsible for supplying glucose for use as a placental fuel and that GT3 is important for glucose transfer to the embryo. (J. Clin. Invest. 1993. 91:845-852.) Key words: deoxyglucose $\bullet$ in situ hybridization • trophoblast • decidua • embryonic development
\end{abstract}

\section{Introduction}

Glucose is the major fuel for energy metabolism and growth in embryogenesis, and yet the mammalian embryo is unable to manufacture this essential substrate until late in development (reviewed in references 1 and 2). Hence, the transfer of glucose from the maternal circulation to the conceptus is a crucial feature of mammalian development. The placenta plays a key role in this transfer process, which appears to be primarily a function of facilitated glucose transport down a concentration gradient from maternal to fetoplacental compartments $(3,4)$. The molecular basis for placental glucose transport and its regulation remain poorly understood at present.

In recent years, there has been rapid progress in elucidating the molecular basis for glucose transport across cell membranes. At least five different facilitative glucose transporters (GTs $1-5,{ }^{1}$ named in order of their cloning) have been charac-

Address correspondence to Jian Zhou, M.D., Ph.D., Building 10, Room 10N262, National Institutes of Health, Bethesda, MD 20892.

Received for publication 5 June 1992 and in revised form 18 September 1992.

The Journal of Clinical Investigation, Inc.

Volume 91, March 1993, 845-852 terized to date (reviewed in references 5 and 6). These integral membrane glycoproteins display significant sequence homology and appear to be members of a genetically related transporter family. The glucose transporter isoforms show unique tissue-specific patterns of distribution and may be subject to differential hormonal regulation (8). For example, GT4 is preferentially expressed in fat and muscle and is regulated by insulin $(7,9)$. GT2 is localized in pancreatic beta cells, hepatocytes, renal proximal tubules, and intestinal epithelium $(6,10)$, while GT5 appears to be expressed primarily in intestinal epithe$\operatorname{lium}(7)$.

To our knowledge, the molecular identity and anatomical disposition of placental glucose transporters has not been previously examined. Since this knowledge may illuminate the mechanisms governing the vectorial transfer of glucose between maternal and fetoplacental compartments, we undertook the mapping of glucose transporter gene expression in the rat uteroplacenta from implantation through term. For this purpose, we used in situ hybridization histochemistry using highly sensitive ${ }^{35} \mathrm{~S}$-labeled cRNA probes complementary to rodent GT 1-4 cDNAs. In addition, since one of the key factors in fetoplacental maternal fuel economy is placental glucose utilization, we used in vivo radiolabeled 2-deoxyglucose uptake to evaluate regional and developmental patterns of placental glucose utilization.

\section{Methods}

Animals and 2-deoxyglucose uptake. Timed pregnant Sprague-Dawley rats were obtained from Taconic Farms, Inc. (Germantown, NY). Uteroplacentas from the 8th-20th $\mathrm{d}$ of gestation were removed from carbon dioxide-anesthetized dams and frozen for later sectioning. Eight rats, on the 14th $\mathrm{d}$ of gestation, received a single intraperitoneal injection of $0.2 \mu \mathrm{Ci} / \mathrm{g}$ 2-deoxy-D-[1- $\left.{ }^{14} \mathrm{C}\right]$ glucose $\left({ }^{14} \mathrm{C}-2 \mathrm{DG}\right)(\mathrm{NEN}$ Dupont, Reston, VA) and were killed in groups of two after 15, 30, 45, and $60 \mathrm{~min}$. Four rats on the 20 th day of gestation were treated in the same fashion. The uteroplacentas were removed and frozen over dry ice. Serial sections ( $10 \mu \mathrm{m}$ in thickness) were cut on a cryostat, thaw mounted onto poly-L-lysine-coated slides, and stored at $-20^{\circ} \mathrm{C}$ until use. The sections from 2-deoxy-D-glucose (2-DG) injected rats were dried and exposed directly to Hyperfilm- $\beta$ Max $^{\mathrm{TM}}$ (Amersham Corp., Arlington Heights, IL) for $2 \mathrm{~d}$.

Riboprobes. GT1 sense and antisense probes were synthesized from a 436-bp Bam H1 fragment corresponding to bases 272-708 of the rat GT 1 cDNA (kindly provided by Dr. M. J. Birnbaum, Harvard Medical School, Boston, MA; reference 11), which was subcloned into pGEM3 for transcription of the antisense probe using T7 RNA polymerase after linearization with HindIII, and for generation of a sense, control probe by SP6 after linearization with EcoR1. The GT2 probe was synthesized from a 418-bp fragment of the rat GT2 cDNA (kindly supplied by Dr. H. F. Lodish, Whitehead Institute for Biomedical Research, MIT, Cambridge, MA; reference 10) corresponding to nucleotides $-18-400$, subcloned into pGEM4, and linearized with EcoR 1 for transcription of antisense with T7. The GT3 probe was synthesized

1. Abbreviations used in this paper: ${ }^{14} \mathrm{C}-2 \mathrm{DG}, 2$-deoxy-D- $\left(1-{ }^{14} \mathrm{C}\right)$ glucose; GT, glucose transporter. 
from a mouse GT3 cDNA fragment, coding for amino acids 207-394, subcloned into pGEM4Z, and transcribed in antisense orientation by T7 after linearization with EcoR I (kindly provided by Dr. G. I. Bell, University of Chicago, Chicago, IL; reference 12). The GT4 probe was synthesized from a 364-bp rat GT4 cDNA fragment corresponding to 95 bases of the $\mathrm{COOH}$-terminal and 269 bases of the $3^{\prime}$ untranslated region (kindly supplied by Dr. M. J. Birnbaum; reference 9 ), subcloned into pGEM4Z, and transcribed with $\mathrm{T} 7$ after linearization with EcoR 1. Northern blots of rat placental RNA were probed with ${ }^{32} \mathrm{P}-\mathrm{la}$ beled GT1 and GT3 riboprobes, and yielded single bands of $\sim 2.8$ and $4.0 \mathrm{~kb}$, respectively (our unpublished data).

In situ hybridization. The synthesis of ${ }^{35} \mathrm{~S}$-labeled $\mathrm{cRNA}$ probes and in situ hybridization protocol has been previously described (13). In brief, cRNA probes were synthesized in $10-\mu 1$ reactions containing $100 \mu \mathrm{Ci}^{35} \mathrm{~S}-\mathrm{CTP}$ and $100 \mu \mathrm{Ci}{ }^{35} \mathrm{~S}-\mathrm{UTP}, 10 \mathrm{mM} \mathrm{NaCl}, 6 \mathrm{mM} \mathrm{MgCl} 2,40$ $\mathrm{mM}$ Tris (pH 7.5), $2 \mathrm{mM}$ spermidine, $10 \mathrm{mM}$ DTT, $500 \mu \mathrm{M}$ each unlabeled ATP and GTP, $25 \mu \mathrm{M}$ each unlabeled UTP and CTP, $500 \mathrm{ng}$ linearized template, $15 \mathrm{U}$ of the appropriate polymerase, and $15 \mathrm{U}$ RNasin (enzymes and other molecular biology reagents were obtained from Promega, Madison, WI). The reaction was incubated at $42^{\circ} \mathrm{C}$ for $60 \mathrm{~min}$, after which the DNA template was removed by digestion with DNase I. Average specific activity of probes generated in this protocol was $2 \times 10^{8} \mathrm{dpm} / \mu \mathrm{g}$.

Before hybridization, sections were warmed to $25^{\circ} \mathrm{C}$, fixed in $4 \%$ formaldehyde, and soaked for $10 \mathrm{~min}$ in $0.25 \%$ acetic anhydride $/ 0.1 \mathrm{M}$ triethanolamine hydrochloride $/ 0.9 \% \mathrm{NaCl}$. Tissue was dehydrated through an ethanol series, delipidated in chloroform, rehydrated, and air dried. ${ }^{35} \mathrm{~S}$-labeled probes were added to hybridization buffer $\left(10^{7}\right.$ $\mathrm{cpm} / \mathrm{ml}$ ) composed of $50 \%$ formamide, $0.3 \mathrm{M} \mathrm{NaCl}, 20 \mathrm{mM}$ Tris $\mathrm{HCl}$, pH 8, 5 mM EDTA, $500 \mu$ g transfer RNA $/ \mathrm{ml}, 10 \%$ dextran sulfate, 10 mM DTT, and $0.02 \%$ each of BSA, ficoll, and polyvinylpyrollidone. Hybridization buffer was added to sections that were incubated under glass coverslips overnight $(14 \mathrm{~h})$ at $55^{\circ} \mathrm{C}$. Slides were washed several times in $4 \times$ standard saline citrate (SSC) to remove cover slips and hybridization buffer, dehydrated, and immersed in $0.3 \mathrm{M} \mathrm{NaCl}, 50 \%$ formamide, $20 \mathrm{mM}$ Tris $\mathrm{HCl}$, and $1 \mathrm{mM}$ EDTA at $60^{\circ} \mathrm{C}$ for $15 \mathrm{~min}$. Sections were then treated with RNase A $(20 \mu \mathrm{g} / \mathrm{ml})$ for $30 \mathrm{~min}$ at $37^{\circ} \mathrm{C}$, passed through graded salts solutions, followed by a 15 -min wash in $0.1 \times \mathrm{SSC}$ at $50^{\circ} \mathrm{C}$. Slides were air dried and apposed to Hyperfilm- $\beta$ Max (Amersham) for $4 \mathrm{~d}$ and then dipped in nuclear emulsion, (NTB3; Kodak, Rochester, NY) stored with desiccant at $4^{\circ} \mathrm{C}$ for $10 \mathrm{~d}$, developed, and stained with hematoxylin and eosin for microscopic evaluation.

Sections from the different gestational age groups were prepared, hybridized, washed, exposed, and developed together to facilitate comparison of signal intensity across the different developmental stages. Figures containing autoradiographs and micrographs comparing different ages represent data acquired in a single experiment. Controls in the form of parallel tissue sections hybridized to a GT1 "sense" probe or pretreated with RNase A were hybridized, washed, and exposed in the same experiments. The background or nonspecific signal from these sections was very minimal.

\section{Results}

Preplacental glucose transporter gene expression. During early pregnancy, there is a robust proliferation of endometrial stromal tissue forming a lush bed for implantation of the blastocyst. GT1 mRNA is extremely abundant in this decidual reaction, while relatively little GT3 mRNA is detected (Fig. 1). In the nonpregnant uterus, GT1 mRNA is detected only in the luminal epithelium and none of the other GT mRNAs are detected (data not shown). The decidua recedes as the embryo grows, with erosion and compression of maternal tissue. Decidual GT1 mRNA is greatly diminished by the time the placenta
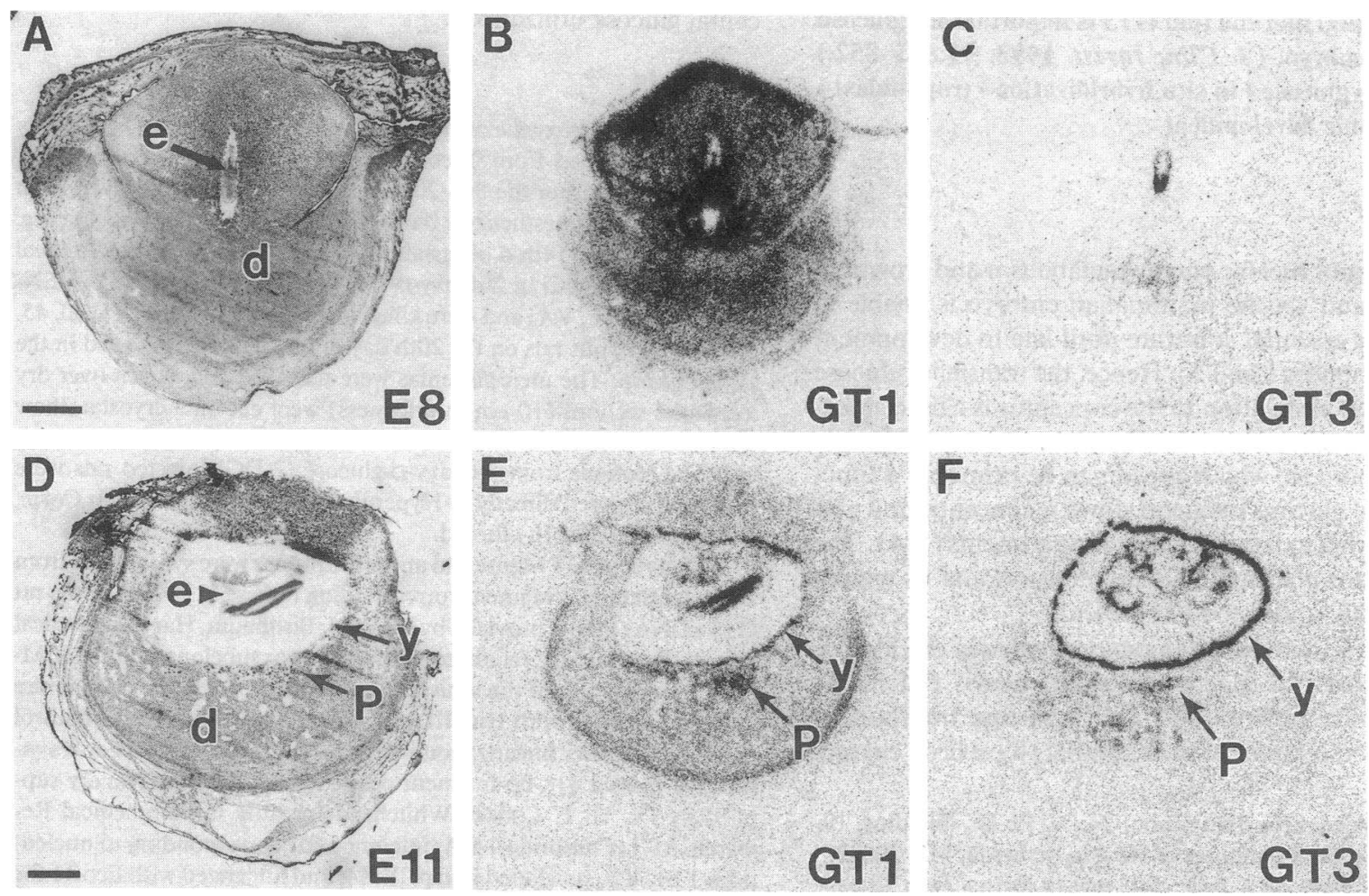

Figure 1. Glucose transporter gene expression in the decidual reaction around the time of implantation $(E 8, A-C)$ and at the time the chorioallantoic placenta begins to develop $(E 11, D-F) . A$ and $D$ show cross-sections of gravid uteri stained with hematoxylin and eosin. $B$ and $E$ and $C$ and $F$ show film autoradiographs of serial sections hybridized to GT1 and GT3 cRNA probes. $e$, Embryo; $P$, placenta; $y$, yolk sac; $d$, decidua. Bar, $1 \mathrm{~mm}$. 

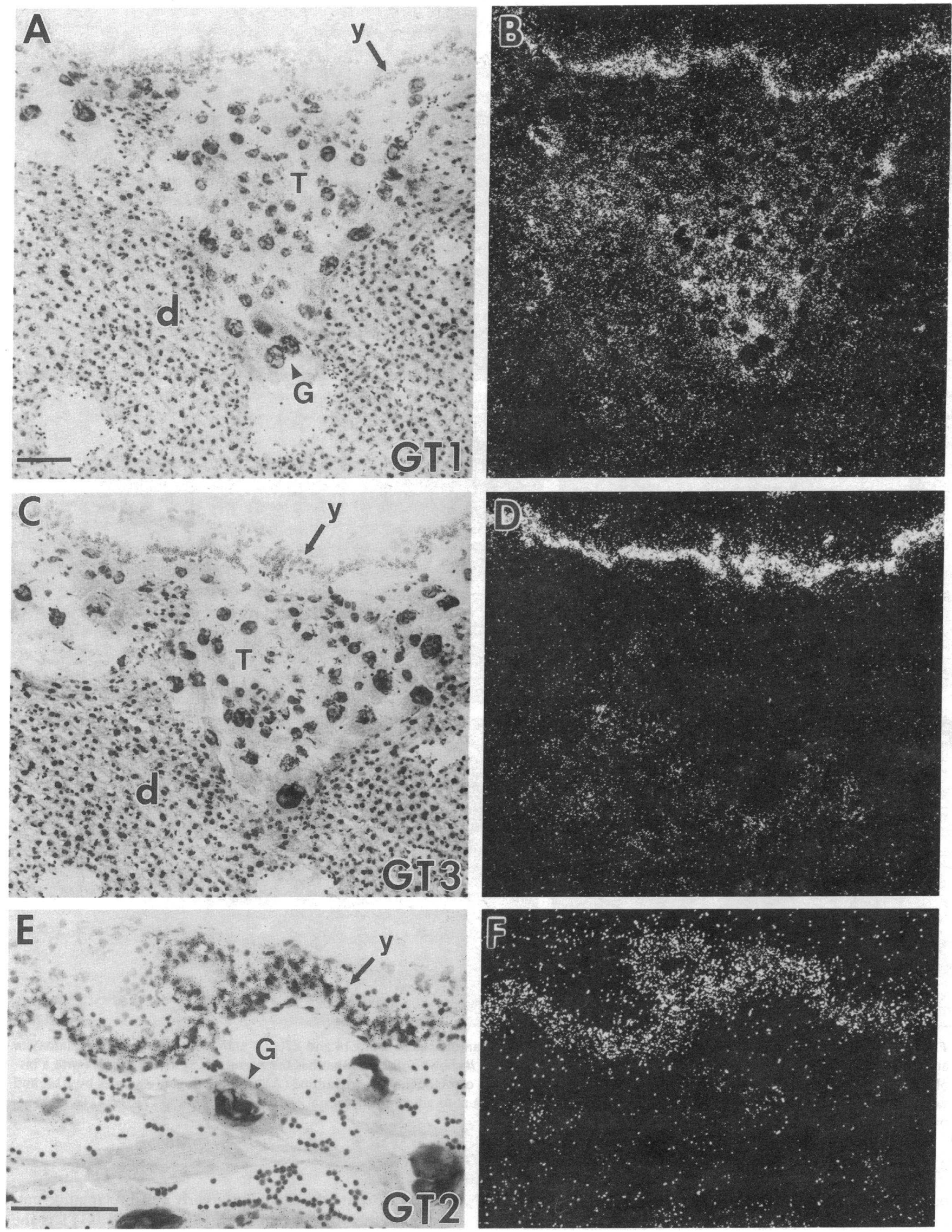

Figure 2. Glucose transporter gene expression in the yolk sac and in trophoblast giant cells encroaching on the decidua. $(A$ and $B)$, ( $C$ and $D)$, and $(E$ and $F$ ) Paired bright and dark field photomicrographs showing GT1, GT3, and GT2 mRNA hybridization, respectively. The trophoblast cells have large metachromatic nuclei and copious cytoplasm that forms a syncitium. Only GT1 mRNA is detected in the trophoblast ( $T$ ). There is a faint signal from the maternal decidua $(d)$ for both GT1 and GT3, while all three GT mRNAs are abundant in the yolk sac $(y)$. Bar, $50 \mu \mathrm{m}$. 

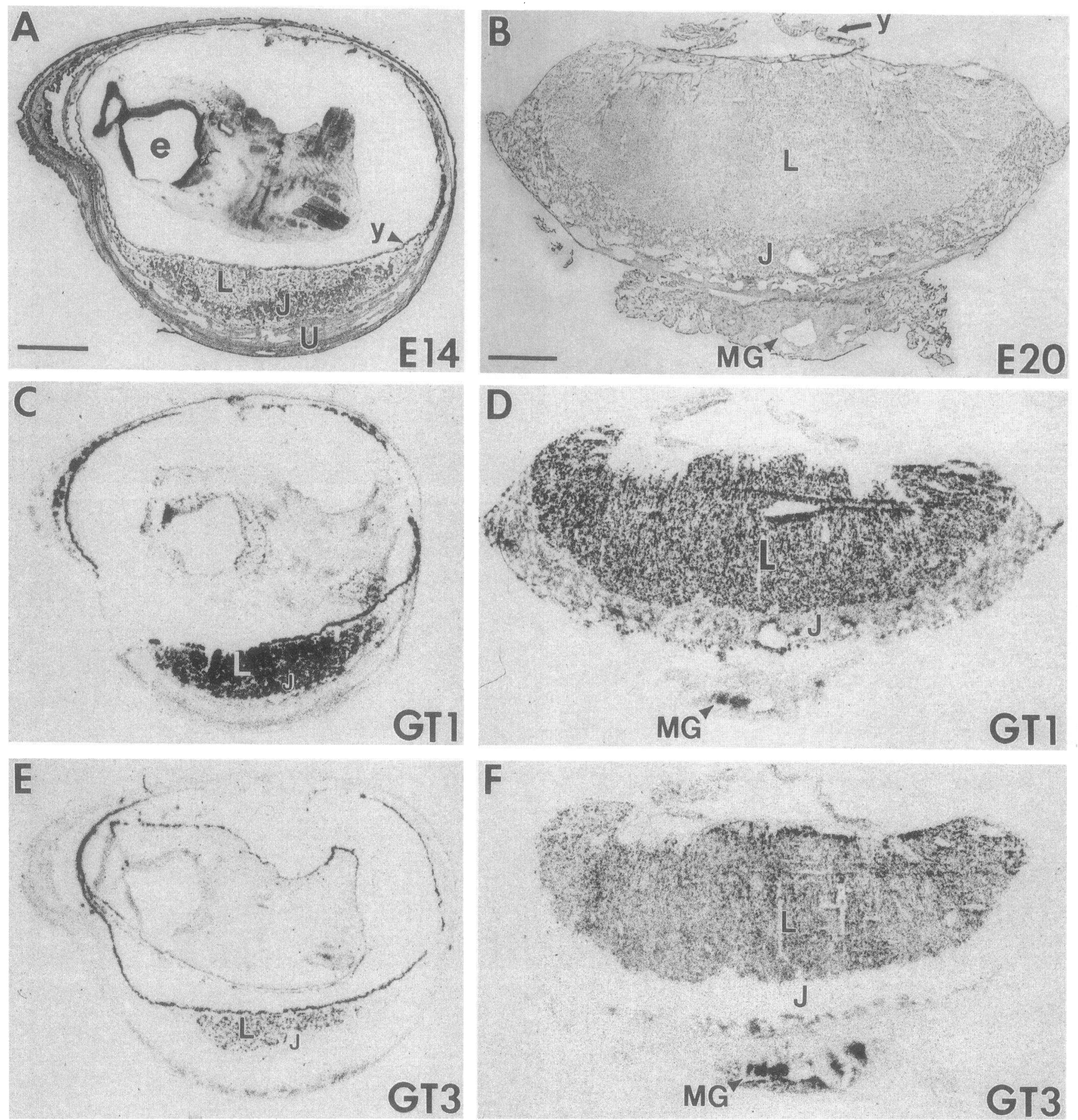

Figure 3. Comparison of GT1 and GT3 gene expression in the chorioallantoic placenta at E14 and E20. $(A$ and $B)$ Photographs of hematoxylin and eosin stained sections; $A$ shows the placenta (and fetus) in situ and $B$ shows a placenta that has been removed from the uterus (with a bit of uterine tissue remaining attached to the base). The placenta consists of well-defined labyrinthine $(L)$ and junctional $(J)$ zones by E14, and expands tremendously in size by E20. $(C$ and $E)$ and $(D$ and $F)$ are film autoradiographs of serial sections hybridized to GT1 or GT3 cRNA probes. $M G$, metrial glands, $U$, uterus. Bar, $2 \mathrm{~mm}$.

begins to form (E11, Fig. $1 D-F)$. Between the time of implantation and the development of the chorioallantoic placenta, the embryonic yolk sac serves important transfer functions. GT1, GT2, and GT3 mRNAs are abundant in the yolk sac (Figs. 1, E and $F$, and 2). GT2 mRNA is not detected elsewhere, and GT4 mRNA is not detected in the reproductive tract or placenta during any stage of gestation. Interestingly, both GT 1 and GT3
mRNAs are quite abundant in the early postimplantation embryo and embryonic membranes (Fig. $1, A-C$ ). GT1 is the predominant transcript in the invading trophoblast (Figs. 1, $E$ and $F$, and $2, A-D$ ).

Glucose transporter gene expression in the chorioallantoic placenta. A mature placental structure composed of two zones is present by $\mathrm{E} 14$ (Fig. $3 \mathrm{~A}$ ). The labyrinthe is the transplacen- 

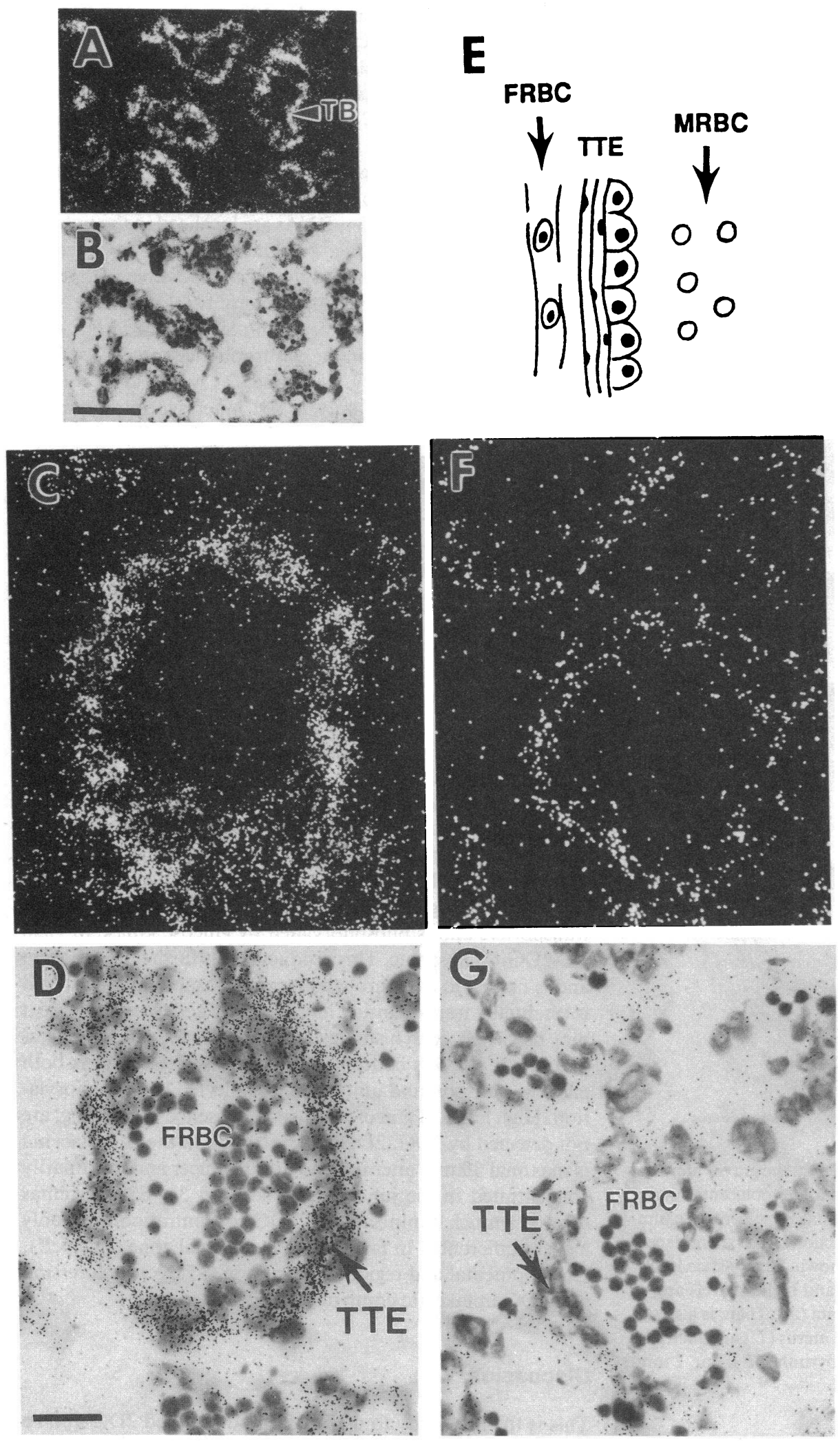

\section{FRBC}

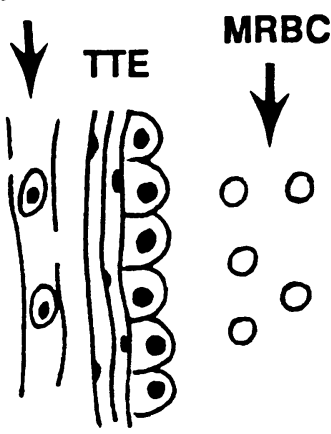

Figure 4. GT1 and GT3 gene expression in the trilaminar trophoblast epithelium. ( $A$ and $B$ ) Paired bright and dark field micrographs showing the trabecular pattern of GT1 mRNA hybridization ( $T B$, trabeculum). ( $C$ and $D$ ) Higher magnification of a cross section through a trabeculuar strand showing GT1 mRNA in the trophoblast epithelium surrounding fetal blood vessels. $(E)$ Diagram illustrating the structure of the trilaminar trophoblast epithelium ( $T T E$ ) interposed between maternal blood and fenestrated fetal capillaries. ( $F$ and $G$ ) GT3 mRNA in the syncitial trophoblast. The nucleated fetal red blood cells $(F R B C)$ are deeply basophilic, while maternal red blood cells $(M R B C)$ do not stain. Bar, $50 \mu$ in $A$ and $B, 200 \mu$ in $C-G$. tal transfer unit in which trabecular cords of fetal (allantoic) blood vessels and mesenchyme, coated with a trilaminar trophoblastic epithelium, are bathed in maternal blood. The junctional zone (also called the basal, reticular, or spongiotropho- blast layer) is the interface between fetal and maternal tissues and is the site of trophoblastic proliferation during placental expansion. It contains clumps of cytotrophoblasts and peripheral giant cells, but only maternal blood vessels and stroma. 


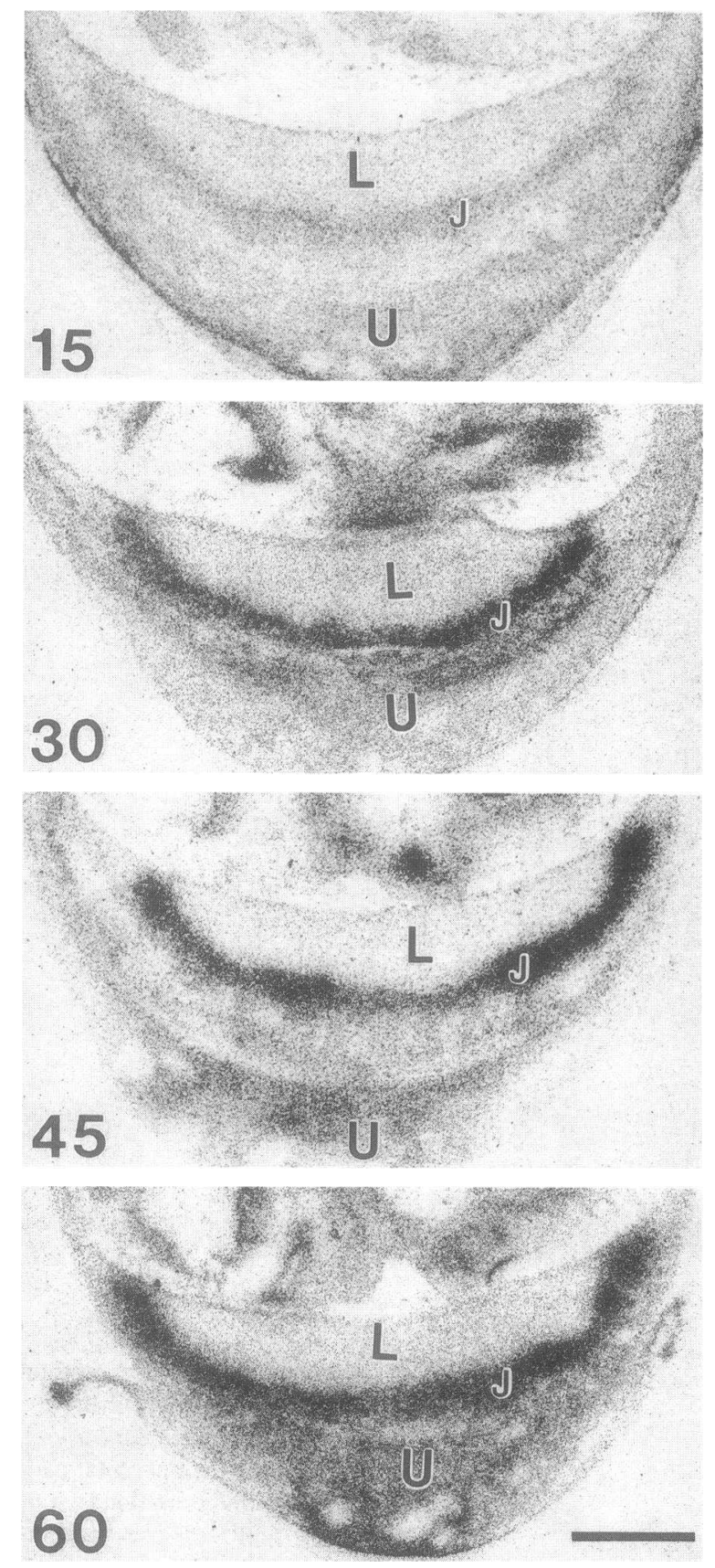

Figure 5. The pattern of 2-deoxyglucose uptake in the gravid uterus of E14 rat. Rats received ${ }^{14} \mathrm{C}-2 \mathrm{DG}(0.2 \mu \mathrm{Ci} / \mathrm{g})$ and uteroplacentas were harvested 15, 30, 45, and $60 \mathrm{~min}$ after injection. Representative film autoradiographs of anatomically matched sections from uteroplacentas obtained at the different time points are shown. Steady-state levels of ${ }^{14} \mathrm{C}$-2DG uptake are apparent by $45 \mathrm{~min}$ and highest levels are concentrated in the junctional zone of placenta $(J)$. There is also significant 2DG concentration in the wall of the uterus $(U)$ and metrial glands. There is little 2DG localized in the labyrinthe $(L)$. Bar, $2 \mathrm{~mm}$.

GT1 mRNA is abundant in both labyrinthine and junctional zones of the E14 placenta (Fig. $3 \mathrm{C}$ ). GT1 mRNA levels decrease between E14 and E20, with the decrease most pronounced in the junctional zone (Fig. 3, $C$ and $D$ ). In contrast to the pattern of GTI expression, GT3 mRNA is detected solely in the placental labyrinth and levels do not change significantly during the course of gestation (Fig. 3, $E$ and $F$ ). Both GT1 and
GT3 mRNAs are concentrated in the uterine metrial glands near the end of pregnancy (Fig. 3, $D$ and $F$ ).

Glucose from the maternal bloodstream must pass through the trilaminar trophoblast epithelium to reach the fenestrated fetal capillaries (Fig. $4 E$ ). Outermost is a fenestrated cytotrophoblast layer beneath which are two syncitiotrophoblast layers that constitute the only barriers to the free diffusion of glucose from maternal to fetal bloodstreams ( reference 14, Fig. $4 E$ ). Both GT1 and GT3 mRNAs are localized in the trabecular syncitiotrophoblast (Fig. 4). These syncitial layers are so thin and closely approximated that it is impossible to differentiate them at the light microscopic level, and, likewise, the isotopic signal cannot be parsed between the different layers. GT1 mRNA is clearly present in the cytotrophoblast as well as syncitiotrophoblast layers (Fig. 4, $C$ and $D$ ), while GT3 mRNA appears to be expressed only in the inner syntiotrophoblast. This distinction cannot be stated unequivocally, given the above-noted limitations in resolution; however, it is supported by the relative differences in abundance in labyrinthine GT1 and GT3 mRNAs. Although it is not standard to compare levels of different $m R N A s$ based on signal intensities generated by different probes, since probe efficiency may vary, in this case, we have internal markers of probe efficiency suggesting that the lower levels of GT3 hybridization signal arising from the labyrinth are not caused by any inherent weakness in the GT3 probe. For example, as seen in Figs. 1 and 2, GT3 mRNA hybridization signal in the yolk sac is higher than that of GT1, and both probes show equal intensity in the uterine metrial glands, while GT1 shows greater signal in the labyrinth than GT3 (Fig. 3, $D$ and $F$ ).

Placental glucose metabolism. Regional and developmental patterns of placental glucose metabolism were evaluated in vivo using the radiolabeled deoxyglucose $\left({ }^{14} \mathrm{C}-2 \mathrm{DG}\right)$ uptake technique (15). The glucose analog, 2-deoxyglucose, competes effectively with glucose for transport via facilitative glucose transporters and is phosphorylated by glucose kinase to yield ${ }^{14} \mathrm{C}-2 \mathrm{DG}-6$-phosphate. This product resists further metabolism and is unable to pass through glucose transport channels, so it is essentially trapped inside metabolically active cells, where it may be visualized in histological sections by film autoradiography. Thus, 2DG is concentrated in cells where there is both efficient transport and utilization, as reflected by phosphorylation. High levels of glucose flux, in the absence of trapping, are not detected by 2DG. 2DG concentration in the E14 placenta is maximal $30 \mathrm{~min}$ after injection of the tracer and is primarily concentrated in the junctional zone (Fig. 5). E20 placentas showed a much diminished ${ }^{14} \mathrm{C}-2 \mathrm{DG}$ concentration, with only a slight differential in favor of the junctional zone (Fig. $6 \mathrm{E}$ ). Developmental and regional patterns of GT1 gene expression and 2DG uptake are compared in Fig. 6.

\section{Discussion}

This is the first study to use in vivo radiolabeled 2DG uptake for the direct examination of placental glucose metabolism. Previous studies have compared the glucose concentration of uterine and umbilical vessels and have shown that the uteroplacenta itself consumes a considerable portion of the glucose destined for the fetoplacental compartment (4), but the anatomical and developmental distribution of placental glucose utilization have not been known. The present study suggests that 

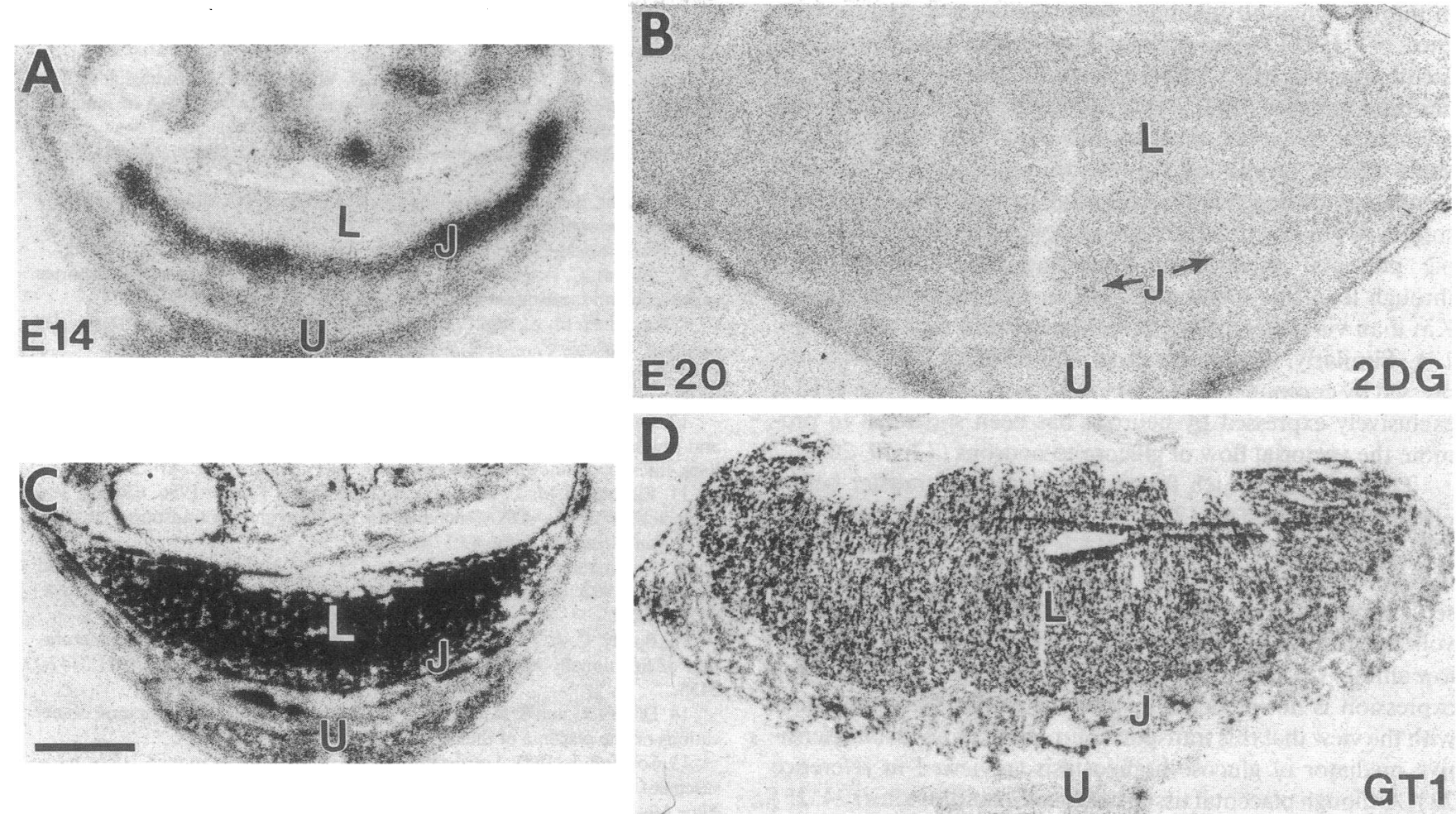

Figure 6. Placental deoxyglucose uptake at E14 and E20 compared with placental GT1 gene expression at the same stages. Bar, $2 \mathrm{~mm}$.

2DG is preferentially concentrated in the junctional zone and is most abundant during the time of rapid placental growth. This zone is the region of cytotrophoblast proliferative and expansionist activity, suggesting that high levels of glucose metabolism may be related to local growth. The low level of 2DG localized in the labyrinthine zone suggests that glucose transport in this region is primarily a function of transfer to the fetus with a relatively low level utilization by labyrinthine cells.

This study has also described the spatiotemporal patterns of glucose transporter gene expression in the developing rat gestation. GT1 gene expression is abundant in tissues demonstrating proliferative growth and high glucose utilization, while GT3 expression is preferentially associated with structures engaged in the transplacental transfer of glucose, with a significant overlap in GT1 and GT3 expression noted in the trophoblast transport membranes. High levels of GT1 mRNA are present in the proliferative endometrium of the peri-implantation decidual reaction and in the junctional zone during the midgestational period of rapid placental growth, associated with high 2DG uptake in this region. GT1 mRNA levels decline in concert with diminishing levels of 2DG uptake, and presumably, a decreased growth rate in the term placenta. These findings are in agreement with the idea that GT1 functions as a constitutive, growth-associated glucose transporter, as suggested by the increased GT1 expression and high glucose metabolism noted in transformed or growth factor-treated cells and established cell lines (5-7).

The anatomical organization of the rat and human placentas are very similar, and both distribute the afferent maternal blood flow first to the junctional zone (in the human, the basal plate) and then to the labyrinthine zone. Hence, the metabolically active cells of the junctional zone have anatomical prior- ity in the reception of maternal glucose, which ensures that the growing placenta will get enough glucose from the maternal circulation to grow large enough to support the gestation. It has been suggested that the placenta consumes glucose "at the expense of the fetus;" however, considering that the placenta is a fetal organ designed for the attachment and nutritional support of the fetus, its growth is clearly central to fetal development.

GT3 gene expression, in contrast to that of GT1, is closely correlated with those structures specialized for transfer of nutrients to the embryo, the yolk sac and the labyrinthine zone of the chorioallantoic placenta. The intensity of GT3 gene expression does not change, but the surface area demonstrating GT3 gene expression increases greatly during the course of gestation, compatible with the necessity for transferring greater amounts of glucose to the growing fetus. It has been calculated that the placental epithelial surface area for exchange of nutrients such as glucose increases by sevenfold during the last week of the rat gestation, associated with a sevenfold increase in fetal weight (16).

GT1 and 3 mRNAs are both expressed in the labyrinthine trophoblast epithelium, which constitutes the membranous barrier between maternal and fetal circulations. It is possible that the two mRNAs are segregated into different layers of syncitiotrophoblast; i.e., GT1 in the intermediate layer and GT3 in the basal layer approximating the fetal circulation. These syncitial layers are so thin and closely apposed that it is difficult to distinguish them, even at the ultrastructural level (14). It is also possible that transporter expression may be asymmetric in individual epithelial cell layers, with one transporter directed to the maternal surface and the other to the fetal surface. Supporting this possibility is the observation that there appears to be a polarization of receptor and enzyme expression 
in human syncitiotrophoblast membranes (reviewed in reference 17). If GT3 were restricted to the fetal side trophoblast membrane and had a higher affinity for glucose than GT1, as has been reported (18), and GT1 located on the maternal side membrane, this anatomical arrangement could function like a valve system, promoting the retention of glucose by the fetal circulation during relative maternal hypoglycemia. Previous studies of glucose transport across isolated human and guinea pig placental membranes have suggested that transport through fetal side membrane does in fact occur with a lower $\mathrm{Km}$ than via maternal side membranes (reviewed in reference 17). Similarly, the observation that GT1 is selectively expressed by components of the blood brain barrier while GT3 is exclusively expressed by neurons has been suggested to promote the vectorial flow of glucose to neurons $(12,19,20)$.

The yolk sac, which functions in nutrient transfer before placental development $(21,22)$, expresses GT2 in addition to GT 1 and 3 mRNAs. GT2 mRNA is not, however, detected in the chorioallantoic placenta. While it is likely that GTs 1 and 3 serve similar roles in maternal fetal glucose transfer in both the yolk sac and the placenta, it is unclear what role the relatively low affinity GT2 isoform might play in the yolk sac. GT4 gene expression is not detected in yolk sac or placenta, consistent with the view that this transporter functions as an insulin-sensitive mediator of glucose homeostasis (reviewed in reference 23 ). Although placental tissue has insulin binding sites $(24,25)$ and insulin receptor mRNA (our unpublished data), placental glucose uptake does not appear to be regulated by insulin (reviewed in reference 26 ).

In summary, this study has shown that GT1 and GT3 appear to be the major placental glucose transporters, and that these two transporters demonstrate distinct anatomical patterns of expression that appear to be functionally significant. GT1 alone is localized in regions of high glucose metabolism and GT3 is localized exclusively in regions devoted to the transplacental transfer of glucose, where there is overlapping GT1 expression. This arrangement suggests that placental GT1 functions to transport glucose from the maternal bloodstream for use as a placental fuel and for further transport via GT3 to the fetal circulation.

\section{Acknowledgments}

We thank Dr. Ian Simpson for helpful discussion and review of the manuscript and Ricardo Dreyfuss for expert photography.

\section{References}

1. Battaglia, F. C., and G. Meschia. 1978. Principal substrates of fetal metabolism. Physiol. Rev. 58:499-527.

2. Jones, C. T. 1991. Control of glucose metabolism in the perinatal period. $J$. Dev. Physiol. 15:81-89.
3. Johnson, L. W., and C. H. Smith. 1985. Glucose transport across the basal membrane of human placental syncitiotrophoblasts. Biochim. Biophys. Acta. 815:44-50.

4. Hay, W. W., Jr., J. W. Sparks, R. B. Wilkening, F. C. Battaglia, and G. Meschia. 1984. Fetal glucose uptake and utilization as functions of maternal glucose concentration. Am. J. Physiol. 246:E237-E242.

5. Mueckler, M. 1990. Family of glucose transporter genes. Diabetes. 39:611.

6. Thorens, B., M. J. Charron, and H. F. Lodish. 1990. Molecular physiology of glucose transporters. Diabetes Care. 13:209-218.

7. Davidson, N. O., Hausman, A. M., If kovits, C. A., Buse, J. B., Gould, G. W., Burant, C. F. and Bell, G. I. 1992. Human intetinal glucose transporter expression and localization og Glut5. Am. J. Physiol. 262:C795-C800.

8. Kasanicki, M. A., and P. F. Pilch. 1990. Regulation of glucose transporter function. Diabetes Care. 13:219-227.

9. Birnbaum, M. J. 1989. Identification of a novel gene encoding an insulinresponsive glucose transporter protein. Cell. 57:305-315.

10. Thorens, B., H. K. Sarkar, H. R. Kaback, and H. F. Lodish. 1988. Cloning and functional expression in bacteria of a novel glucose transporter present in liver, intestine, kidney and beta-pancreatic islet cells. Cell. 55:281-290.

11. Birnbaum, M. J., H. C. Haspel, and O. M. Rosen. 1986. Cloning and characterization of a cDNA encoding the rat brain glucose transporter protein. Proc. Natl. Acad. Sci. USA. 83:5784-5788.

12. Nagamatsu, S., J. M. Kornhauser, C. F. Burant, S. Seino, K. E. Mayo, and G. I. Bell. 1992. Glucose transporter expression in the brain. J. Biol. Chem. 267:467-472.

13. Bondy, C. A. 1991. Transient IGF-I gene expression during the maturation of functionally-related central projection neurons. J. Neurosci. 11:34423455 .

14. Davies, J., and S. R. Glasser. 1968. Histological and fine structural observations on the placenta of the rat. Acta. Anat. 69:542-608.

15. Sokoloff, L. 1981. Localization of functional activity in the CNS by measurement of glucose utilization with radioactive deoxyglucose. J. Cereb. Blood Flow Metab. 1:7-36.

16. Shelley, H. J. 1979. Transfer of carbohydrate. In Placental Transfer, Eds G. V. P. Chamberlain and A. W. Wilkinson, editors. Pitman Medical Publishing Co., Kent, England. 118-141.

17. Yudilevich, D. L., and C. P. D. Wheeler. 1988. Placental transport. In Fetal and Neonatal Development. C. T. Jones, editor. Perinatology Press, Ithaca, NY. 47-57.

18. Gould, G. W., H. M. Thomas, T. J. Jess, and G. I. Bell. 1991. Expression of human glucose transporters in Xenopus oocytes: kinetic characterization and substrate specificities of the erythrocyte, liver and brain isofroms. Biochemistry. 30:5139-5145.

19. Maher, F., T. M. Davies-Hill, P. G. Lysko, R. C. Henneberry, and I. A. Simpson. 1991. Expression of two glucose transporters, GLUT1 and GLUT3, in cultured cerebellar neurons: evidence for neuron-specific expression of GLUT3. Mol. Cell. Neurosci. 2:351-360.

20. Bondy, C. A., W.-H. Lee, and J. Zhou. 1992. Ontogeny and cellular distribution of brain glucose transporter gene expression. Mol. Cell. Neurosci. 3:305-314.

21. Noer, H. R., and H. W. Mossman. 1947. Surgical investigation of the function of the inverted yolk sac placenta in the rat. Anat. Rec. 98:31-37.

22. Hard, W. L. 1946. Histochemical and quantitative study of phosphatase in the placenta and fetal membranes of the guinea pig. Am. J. Anat. 78:47-77.

23. Kahn, B. K. 1992. Facilitative glucose transporters. Regulatory mechanisms and dysregulation in diabetes. J. Clin. Invest. 89:1367-1374.

24. Posner, B. I. 1974. Insulin receptors in human and animal placental tissue. Diabetes. 23:209-217.

25. Nelson, D. M., R. M. Smith, and L. Jarett. 1978. Non-uniform distribution and grouping of insulin receptors on the surface of the human syncitiotrophoblast. Diabetes. 27:530-538.

26. Ingermann, R. I. 1987. Control of placental glucose transfer. Placenta. $8: 557-571$ 